\title{
BMJ Open Pneumococcal pneumonia and carriage in Africa before and after introduction of pneumococcal conjugate vaccines, 2000-2019: protocol for systematic review
}

To cite: Kalata NL, Nyazika TK, Swarthout TD, et al. Pneumococcal pneumonia and carriage in Africa before and after introduction of pneumococcal conjugate vaccines, 2000-2019: protocol for systematic review. BMJ Open 2019;9:e030981. doi:10.1136/ bmjopen-2019-030981

- Prepublication history for this paper is available online. To view these files, please visit the journal online (http://dx.doi. org/10.1136/bmjopen-2019030981).

\section{NLK and TKN contributed} equally.

NLK and TKN are joint first authors.

Received 10 April 2019 Revised 18 October 2019 Accepted 21 October 2019

Check for updates

(C) Author(s) (or their employer(s)) 2019. Re-use permitted under CC BY-NC. No commercial re-use. See rights and permissions. Published by BMJ.

For numbered affiliations see end of article.

Correspondence to Mr Tinashe K. Nyazika; tknyazika@gmail.com

\section{ABSTRACT}

Introduction Africa harbours a high burden of pneumococcal disease, with associated high mortality rates. Despite 34 countries introducing the pneumococcal conjugate vaccine, which reduces the risk of pneumococcal carriage (a prerequisite for disease) of some of the most pathogenic pneumococcal serotypes, it remains uncertain whether they will achieve the sustained direct or indirect protection necessary to reduce pneumococcal carriage to levels sufficient to interrupt transmission and disease. We will therefore summarise the available data on the impact of the pneumococcal conjugate vaccine in reducing vaccine serotype carriage and pneumococcal pneumonia in Africa between 2000 and 2019.

Methods and analysis Using a predetermined search strategy, we will conduct a comprehensive search of PubMed, MEDLINE database, the Excerpta Medica Database, the ISI Web of Science (Science Citation Index), Scopus and the African Index Medicus to identify published studies reporting the prevalence of Streptococcus pneumoniae carriage (vaccine type and non-vaccine type), incidence rates of pneumococcal pneumonia and mortality among children, adults and HIV-infected (allages) pre-pneumococcal conjugate vaccine (PCV) and post-PCV introduction (published between 1st January 2000 and 31st December 2019) in African countries that have introduced PCVs (PCV7/PCV10/PCV13) in their routine national immunisation programme. The studies retained and data extracted will be assessed for bias using prevalidated tools and checklists. Heterogeneity across studies will be assessed using the $\chi^{2}$ test on Cochrane $Q$ statistic. A random effect meta-analysis will be used to estimate the overall prevalence of pneumococcal carriage and incidence of pneumococcal pneumonia across studies with similar characteristics. Results will be reported in compliance with the Meta-Analysis Of Observational Studies in Epidemiology guidelines. The protocol has been prepared in accordance to the 2015 guidelines on Preferred Reporting Items for Systematic Reviews and Meta-Analyses.
Strengths and limitations of this study

To the best of our knowledge, this study will be the first systematic review that will comprehensively compare and explore the impact of pneumococcal conjugate vaccine (PCV) on carriage, pneumococcal pneumonia (invasive and non-invasive) and mortality associated with the disease prevaccine and postvaccine introduction in adults and other at risk groups living in Africa.

- Small number of studies eligible across regions could impact the quality of our estimates.

- Presence of high heterogeneity across studies, particularly in countries where PCV7, PCV10 or PCV13 were in use around the same time and were serotyping was only done for vaccine-type pneumococcal strains.

Ethics and dissemination This systematic review will not require ethical approval as we will be using already published data. The final manuscript will be submitted for publication in a peer-reviewed journal and presented at conferences.

PROSPERO registration number CRD42019130976.

\section{INTRODUCTION}

Streptococcus pneumoniae ('the pneumococcus') is a common coloniser of the nasopharynx and a major cause of morbidity and mortality among children under the age of 5 years worldwide. ${ }^{2}$ High rates of pneumococcal disease are found in Africa and other low-income and middle-income countries, which harbour an accumulation of factors conducive for high and persistent pneumococcal carriage and transmission including, high-density living conditions, poverty and high HIV seroprevalence. ${ }^{3-5}$ In 2015 , pneumococcal pneumonia accounted for $55.4 \%$ 
(95\% uncertainty interval: 31.5-79.1) of lower respiratory deaths in all ages. ${ }^{1}$ Implementation of treatment strategies, which focus on early detection and antimicrobial therapy of suspected pneumonia cases, has been difficult in Africa. ${ }^{6}$ Therefore, introduction of an effective vaccine against pneumococcal carriage for children is crucial in this region as carriage is a prerequisite for disease.

With support from Gavi (the Vaccine Alliance), 34 African countries introduced the pneumococcal conjugate vaccines (PCV7/PCV10/PCV13) into their extended immunisation programmes between 2009 and $2015^{78}$ in line with WHO recommendations. ${ }^{9}$ These vaccines have been shown to be highly effective in reducing both incidence of pneumococcal disease and prevalence of carriage by some of the most pathogenic of the 97 known pneumococcal serotypes. ${ }^{10}$ Furthermore, the vaccine indirectly protects the PCV-unvaccinated population against pneumococcal carriage and disease by reducing pneumococcal transmission from PCV-vaccinated children who are at greater risk of carriage and, therefore, sources of transmission. ${ }^{2}{ }^{11-13}$ However, PCV's indirect protection on other high-risk populations (including HIV-infected individuals) remains to be seen in Africa. ${ }^{11}{ }^{14}$ Studies conducted in Africa have demonstrated high nasopharyngeal carriage of pneumococcus among the HIV-infected adults on antiretroviral therapy (ART), with estimated point prevalence ranging between $40 \%$ and $80 \% .^{12} 15$ Therefore, it remains uncertain whether the introduction of PCV in Africa will achieve the sustained direct or indirect protection necessary to reduce pneumococcal carriage to levels sufficient to interrupt transmission and disease. $^{16}$

Systematic reviews that have been done so far on the impact of PCV in Africa have focused on carriage and clinical pneumonia endpoints in children. ${ }^{17}{ }^{18}$ None have summarised these data in the adult or important at-risk populations like HIV-infected individuals. We therefore plan to synthesise the available published data and update the evidence on the impact of the PCV introduction on pneumococcal carriage, pneumococcal pneumonia and associated mortality in Africa between 2000 and 2019.

\section{OBJECTIVES}

\section{General objectives}

1. To summarise available data on the impact of the PCV on the prevalence of pneumococcal carriage (vaccine type - VT and non-vaccine type - NVT) and its impact on pneumococcal pneumonia and associated mortality in Africa between 2000 and 2019.

\section{Specific objectives}

Using data published between 2000 and 2019, this review will assess the impact of PCV by:

1. Comparing the trend in pneumococcal carriage prevalence in Africa before and after PCV introduction.

2. Comparing the trends in the incidence and/or prevalence of pneumococcal pneumonia (invasive and noninvasive) in Africa before and after PCV introduction.
3. Estimating the mortality associated with pneumococcal pneumonia in Africa.

\section{METHODS}

Information sources, search strategy and study identification

We will conduct a comprehensive literature search in PubMed, MEDLINE database, the Excerpta Medica Database, the ISI Web of Science (Science Citation Index), Scopus and the African Index Medicus to identify all studies published between 1st January 2000 to 31st December 2019, meeting our inclusion and exclusion criteria using the literature search strategy outlined in table 1. Using a structured screening guide, we will screen the reference lists for eligible articles and relevant reviews as well as conference abstracts to identify additional sources of information. Search results will be compiled and managed using the EndNote X9 citation software.

\section{Eligibility criteria of relevant studies}

Inclusion criteria

\section{Population}

Children (0-15 years), adults (15-64 years), the elderly (>65years) and HIV-infected individuals (all ages) residing in African countries.

\section{Intervention}

PCVs (PCV 7, PCV10 and PCV13) introduction into the country's routine infant immunisation programmes.

\section{Comparators}

Ten-year period (2000-2010) before PCV introduction versus 10-year period (2011-2019) after PCV introduction.

\section{Outcomes}

Percentage difference in the pooled point prevalence of pneumococcal carriage post when compared with pre-PCV introduction; adjusted prevalence ratios in the pooled prevalence of pneumococcal pneumonia between post-PCV and pre-PCV introduction; and adjusted mortality rate ratios for post-PCV and pre-PCV introduction.

\section{Type of studies}

We will include randomised control trials, non-randomised trials and observational studies reporting prevalence of pneumococcal carriage and/or incidence rates for disease and/or deaths and were published between 1st January 2000 and 31st December 2019 without language restriction.

\section{Serotyping methods}

For pneumococcal carriage, identification and serotyping of $S$. pneumoniae must be from a pernasal swab using culture (as per WHO guidelines, ${ }^{19}$ PCR (lyt-A gene amplification), microarray or based on whole genome sequencing (WGS). 


\begin{tabular}{|c|c|}
\hline Search & Search terms and combinations \\
\hline 1 & ("Pneumococc"” OR "Strep* pneumo" OR "S. pneumo*” OR “Streptococc*”) \\
\hline 3 & 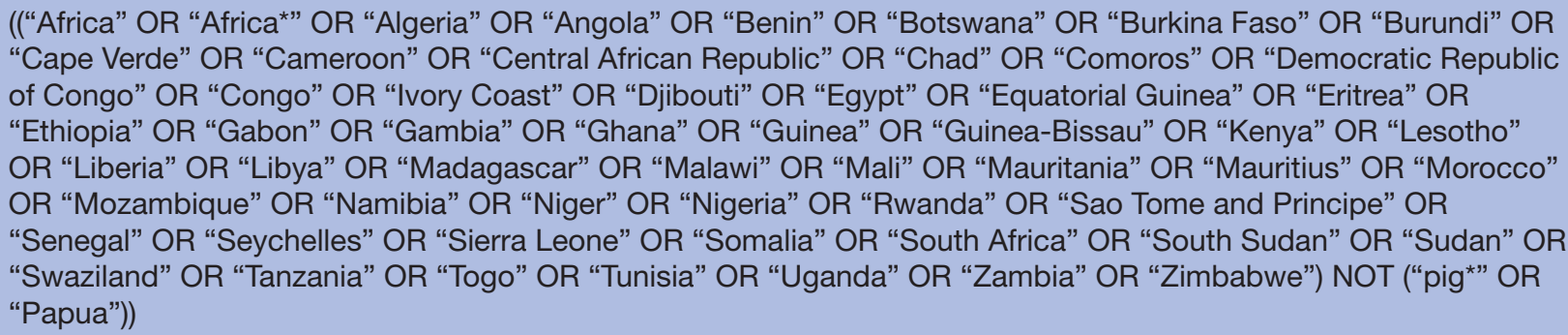 \\
\hline 6 & ("mortality rate" OR “case fatality" OR "death rate") \\
\hline 7 & $\begin{array}{l}\text { Carriage: } \\
\text { \#1 AND \#2 AND \#3 AND \#4 }\end{array}$ \\
\hline 8 & $\begin{array}{l}\text { Pneumococcal pneumonia and associated mortality: } \\
\# 1 \text { AND \#2 AND \#3 AND (\#5 AND/OR \#6) }\end{array}$ \\
\hline 9 & Filters: Publication date from 2000/01/01 to 2019/12/31; Humans \\
\hline
\end{tabular}

\section{Pneumococcal pneumonia}

For a pneumococcal pneumonia diagnosis among adults, a minimum of either (1) symptoms consistent with an acute chest infection (at least cough or dyspnoea) or (2) new infiltrates on radiograph and (3) pneumococcal antigen detection in either blood, urine or pleural fluid. For children (1-15 years), the case definition is the criteria for adults plus symptoms of fast breathing, chestindrawing and nasal flaring. These definitions have been adopted in order to increase the specificity of identifying pneumococcal pneumonia but are limited in their sensitivity.

\section{Study exclusion criteria}

1. Any study reporting impact data of vaccines within 12 months of PCV introduction into routine extended programme of immunisation and data reported in such a way that impact cannot be defined without data from within 12 months of PCV introduction.

2. Studies with small sample size (less than 50 participants), letters, commentaries, narratives and editorials, will be excluded as they are likely to have biased estimates, and this could affect our estimates.

3. Studies that did not distinguish between pre-PCV and post-PCV introduction periods in the data presented.

\section{Data extraction}

Three independent reviewers (NLK, TKN and TDS) will assess the articles for inclusion based on a predetermined inclusion and exclusion criteria. Those included after initial screening will be further evaluated for methodological quality and presence of bias. Discordance will be resolved by discussion between reviewers to reach consensus or by a fourth member of the team (KJ). All articles retrieved will be stored and managed using Endnote X9 throughout the review process. A standardised data extraction form designed specifically for each outcome of interest, will be used to collect information on the following parameters:

1. Study identification: name of first author, year of publication, year of participant inclusion, country, type of publication, language of publication.

2. Study characteristics: study design (eg, cross-sectional, cohort, case control, clinical trial), setting (hospital, outpatient, population, institution (eg, school or care facility), urban/rural), period of surveillance/recruitment, sample size, age (mean or median, range), proportion of HIV-infected participants and method of confirmation (if any), proportion on ART (if any), proportion PCV-vaccinated (if any), proportion with natural pneumococcal carriage (VT, NVT, total), diagnostic method for pneumococcal carriage detection and serotyping (culture, PCR, DNA microarray, WGS), diagnostic criteria for pneumococcal pneumonia, duration of follow-up for cohort studies and mortality rates.

3. Epidemiological estimates of pneumococcal carriage: prevalence of VT and NVT carriage.

4. Epidemiological estimates for pneumococcal pneumonia: prevalence and/or incidence of pneumococcal pneumonia and/or mortality in all subpopulations, where reported (infants below age of 1 year, children 1-4 years, children 5-15 years and adults $16+$ years), the elderly and HIV infected. When estimates are not 
available or cannot be computed, the corresponding authors will be contacted to request for any missing information of these estimates.

\section{Appraisal of quality of reporting and the risk of bias}

We will assess the quality of reporting of the studies using either the Strengthening the Reporting of Observational studies in Epidemiology or the Consolidated Standards of Reporting Trials checklist depending on the nature of the study (observational study or clinical trial).$^{20-22}$

We will use the 10-item risk of bias tool for prevalence studies developed by Hoy $e t a l^{23}$ to assess the risk of bias for all the studies included using the full text publications. Bias risk scores will be presented in a table and inter-rater agreement will be assessed using a weighted Cohen's kappa statistic. ${ }^{24} 25$

\section{Data analyses and reporting}

We will use the metaprop command provided within the STATA software V.15 to analyse the data. ${ }^{26}$ Heterogeneity of studies with similar study characteristics will be evaluated by the $\chi^{2}$ test on Cochrane's $Q$ statistic ${ }^{27}$ and quantified using $\mathrm{I}^{2}$ values assuming that $\mathrm{I}^{2}$ of $25 \%$, $50 \%$ and $75 \%$ represent low, medium and high heterogeneity, respectively. ${ }^{28}$ Study-specific estimates will be pooled through a random effect meta-analysis to obtain an overall summary estimate of the prevalence of S. pneumoniae carriage, incidence of pneumococcal pneumonia and mortality after stabilising the variance of individual studies using the Freeman-Tukey double arcsine transformation. ${ }^{29}$ For impact of PCV on carriage, the percentage change in point prevalence will be calculated to compare pooled prevalence estimates in the before and after PCV introduction periods. Mortality rate ratios will be calculated to compare pooled mortality rates for the periods before and after PCV introduction. Meta-analysis results will be presented on a forest plot. Visual analysis of funnel plot and Egger's test will be done to detect small study effect. ${ }^{30} 31$ All tests will be two-sided and statistical significance will be defined as $p<0.05$. In case of marked heterogeneity, a descriptive analysis will be done instead of a meta-analysis.

We will report our results according to the MetaAnalysis Of Observational Studies in Epidemiology guidelines. ${ }^{32}$ The process with which studies were selected will be summarised using a flow diagram. Reasons for study exclusion will be described and quantitative data will be presented in summary tables and graphs (for trends) where appropriate.

\section{Ethics and dissemination}

This systematic review will not require ethical approval as we will be using already published data. The findings will be summarised in a manuscript and submitted in peerreviewed journals and presented at relevant conferences.

\section{Patient and public involvement}

This systematic review will use published scientific data and will not involve patients or members of the public.

\section{Author affiliations}

${ }^{1}$ Malawi-Liverpool-Wellcome Trust Clinical Research Programme, University of Malawi College of Medicine, Blantyre, Malawi

${ }^{2}$ Department of Clinical Sciences, Liverpool School of Tropical Medicine, Liverpool, UK

${ }^{3}$ The Queens Medical Research Institute, University of Edinburgh, Edinburgh, UK

${ }^{4}$ Centre for Global Vaccine Research, Institute of Infection and Global Health,

Liverpool, UK

${ }^{5}$ Division of Infection and Immunity, University College London, London, UK

Acknowledgements The Malawi-Liverpool-Wellcome Trust Clinical Research Programme (MLW) receives a strategic grant from the Wellcome Trust (UK).

Contributors NLK, TKN, KJ and TDS conceived the study and drafted the manuscript. The manuscript was revised by DE, SBG, NF, RSH and KJ. All authors approved the final version of the manuscript. TKN is the guarantor of the manuscript.

Funding The authors have not declared a specific grant for this research from any funding agency in the public, commercial or not-for-profit sectors.

Competing interests None declared.

Patient consent for publication Not required.

Provenance and peer review Not commissioned; externally peer reviewed.

Open access This is an open access article distributed in accordance with the Creative Commons Attribution Non Commercial (CC BY-NC 4.0) license, which permits others to distribute, remix, adapt, build upon this work non-commercially, and license their derivative works on different terms, provided the original work is properly cited, appropriate credit is given, any changes made indicated, and the use is non-commercial. See: http://creativecommons.org/licenses/by-nc/4.0/.

ORCID iD

Tinashe K. Nyazika http://orcid.org/0000-0003-0367-4265

\section{REFERENCES}

1 Troeger C, Forouzanfar M, Rao PC, et al. Estimates of the global, regional, and national morbidity, mortality, and aetiologies of lower respiratory tract infections in 195 countries: a systematic analysis for the global burden of disease study 2015. Lancet Infect Dis 2017;17:1133-61.

2 Heinsbroek E, Tafatatha T, Phiri A, et al. Pneumococcal carriage in households in Karonga District, Malawi, before and after introduction of 13-valent pneumococcal conjugate vaccination. Vaccine 2018;36:7369-76.

3 Vos T, Barber RM, Bell B, et al. Global, regional, and national incidence, prevalence, and years lived with disability for 301 acute and chronic diseases and injuries in 188 countries, 1990-2013: a systematic analysis for the global burden of disease study 2013 . The Lancet 2015;386:743-800.

4 Melegaro A, Gay NJ, Medley GF. Estimating the transmission parameters of pneumococcal carriage in households. Epidemiol Infect 1999;132:433-41.

5 Hill PC, Townend J, Antonio M, et al. Transmission of Streptococcus pneumoniae in rural Gambian villages: a longitudinal study. Clin Infect Dis 2010;50:1468-76.

6 Pio A. Standard case management of pneumonia in children in developing countries: the cornerstone of the acute respiratory infection programme. Bull World Health Organ 2003;81:298-300.

7 Loharikar A, Dumolard L, Chu S, et al. Status of new vaccine introduction - worldwide, September 2016. MMWR Morb Mortal Wkly Rep 2016;65:1136-40.

8 Wang SA, Mantel AF, Dobo MG. Progress in introduction of pneumococcal conjugate vaccine-worldwide, 2000-2013. Morb Mortal Wkly Rep 2013;62:308-11.

9 WHO Publication. Pneumococcal vaccines WHO position paper 2012 - recommendations. Vaccine 2012;30:4717-8.

10 Chaguza C, Cornick JE, Andam CP, et al. Population genetic structure, antibiotic resistance, capsule switching and evolution of invasive pneumococci before conjugate vaccination in Malawi. Vaccine 2017;35:4594-602.

11 Verani JR, Massora S, Acácio S, et al. Nasopharyngeal carriage of Streptococcus pneumoniae among HIV-infected and -uninfected children. Plos One 2018;13:e0191113.

12 Heinsbroek E, Tafatatha T, Chisambo C, et al. Pneumococcal acquisition among infants exposed to HIV in rural Malawi: a longitudinal household study. Am J Epidemiol 2016;183:70-8. 
13 Lexau CAet al. Changing epidemiology of invasive pneumococcal disease among older adults in the era of pediatric pneumococcal conjugate vaccine. JAMA 2005;294:2043-51.

14 Bonten MJM, Huijts SM, Bolkenbaas M, et al. Polysaccharide conjugate vaccine against pneumococcal pneumonia in adults. $N$ Engl J Med 2015;372:1114-25.

15 Swarthout TD, Fronterre C, Lourenço J, et al. High residual prevalence of vaccine serotype Streptococcus pneumoniae carriage 4 to 6 years after introduction of 13-valent pneumococcal conjugate vaccine in Malawi: a prospective serial cross-sectional study. bioRxiv 2018.

16 Olayinka F, Ewald L, Steinglass R. Beyond new vaccine introduction: the uptake of pneumococcal conjugate vaccine in the African region. Pan Afr Med J 2017;27:1-4.

17 Alicino C, Paganino C, Orsi A, et al. The impact of 10-valent and 13-valent pneumococcal conjugate vaccines on hospitalization for pneumonia in children: a systematic review and meta-analysis. Vaccine 2017;35:5776-85

18 Ngocho JS, Magoma B, Olomi GA, et al. Effectiveness of pneumococcal conjugate vaccines against invasive pneumococcal disease among children under five years of age in Africa: a systematic review. PLoS One;14:e0212295.

19 Satzke C, Turner P, Virolainen-Julkunen A, et al. Standard method for detecting upper respiratory carriage of Streptococcus pneumoniae: Updated recommendations from the World Health Organization Pneumococcal Carriage Working Group. Vaccine 2013;32:165-79.

20 von Elm E, Altman DG, Egger M, et al. Strengthening the reporting of observational studies in epidemiology (STROBE) statement: guidelines for reporting observational studies. BMJ 2007;335:806-8.

21 Schulz KF, Altman DG, Moher D, et al. Consort 2010 statement: updated guidelines for reporting parallel group randomised trials. BMJ 2010;340.
22 Turner L, Shamseer L, Altman DG, et al. Consolidated standards of reporting trials (CONSORT) and the completeness of reporting of randomised controlled trials (RCTs) published in medical journals. In: Cochrane methodology review group, editor. Cochrane database Syst Rev. 5, 2012.

23 Hoy D, Brooks $P$, Woolf A, et al. Assessing risk of bias in prevalence studies: modification of an existing tool and evidence of interrater agreement. J Clin Epidemiol 2012;65:934-9.

24 Cohen J. Weighted kappa: nominal scale agreement provision for scaled disagreement or partial credit. Psychol Bull 1968;70:213-20

25 McHugh ML. Interrater reliability: the kappa statistic. Biochem Med 2012:276-82.

26 Nyaga VN, Arbyn M, Aerts M. Metaprop: a Stata command to perform meta-analysis of binomial data. Arch Public Health $2014 ; 72$.

27 Huedo-Medina TB, Sánchez-Meca J, Marín-Martínez F, et al. Assessing heterogeneity in meta-analysis: $Q$ statistic or $\mathrm{I}^{2}$ index? Psychol Methods 2006;11:193-206.

28 Delgado-Rodríguez M, Sillero-Arenas M. Systematic review and meta-analysis. Med Intensiva 2018;42:444-53.

29 White IR. Multivariate Random-effects meta-analysis. Stata J 2009;9:40-56.

30 Sterne JAC, Egger M. Funnel plots for detecting bias in metaanalysis: guidelines on choice of axis. J Clin Epidemiol 2001;10.

31 Sterne JAC, Sutton AJ, loannidis JPA, et al. Recommendations for examining and interpreting funnel plot asymmetry in meta-analyses of randomised controlled trials. BMJ 2011;343:d4002.

32 Stroup DF, Berlin JA, Morton SC, et al. Meta-Analysis of observational studies in epidemiology: a proposal for reporting. meta-analysis of observational studies in epidemiology (moose) group. JAMA 2000;283:2008-12. 